\title{
Fundamental Limitations in Self-Sensing Magnetic Bearings when Modeled as Linear Periodic Systems
}

\author{
K.S. Peterson and R.H. Middleton and J.S. Freudenberg
}

\begin{abstract}
In "Magnetic Bearing Measurement Configurations and Associated Robustness and Performance Limitations", Thibeault and Smith demonstrate that self-sensing magnetic bearings are impractical due to fundamental limitations in the achievable closed-loop robustness. Due to experimental data which appeared to contradict these results, Maslen, Montie, and Iwasaki showed that significantly better robustness is achievable in "Robustness limitations in self-sensing magnetic bearings" if the magnetic bearing is modeled as a linear periodic (LP) system rather than the linear time invariant (LTI) system used by Thibeault and Smith. The present paper explores why modeling the self-sensing magnetic bearing as a LP system improves the achievable robustness. This is accomplished by utilizing lifting to analyze the LP model as a MIMO discrete LTI system.
\end{abstract}

\section{INTRODUCTION}

By suspending the rotating shaft, or rotor, between opposing sets of electromagnets, magnetic bearings are capable of providing non-contacting support as shown in Fig. 1. This allows magnetic bearings to eliminate concerns that arise from friction, wear, and lubrication in high-speed applications. Furthermore, the electromagnets can provide active damping and the absence of lubricants allows for the magnetic bearing to operate in isolation from the environment. Unfortunately, magnetic bearings are inherently unstable and thus require closed-loop control for stable operation.

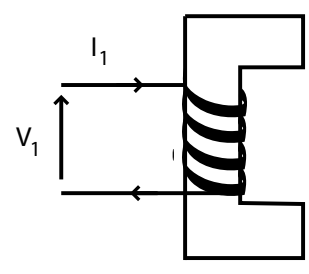

Left Electromagnet
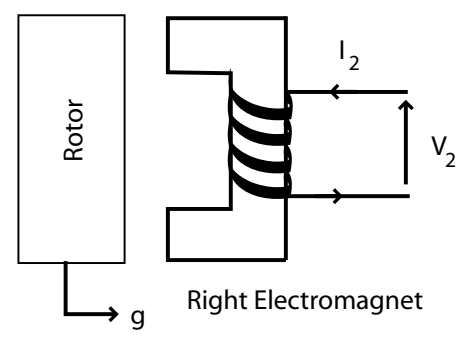

Fig. 1. 1-dimensional magnetic bearing model.

Self-sensing magnetic bearings are those which use feedback based on the measured current alone. Measuring current for use in the feedback is preferable as current is considerably less expensive to measure than rotor position. Based on the analysis of a self-sensing magnetic bearing as a linear time invariant (LTI) system, Thibeault and Smith [13] showed that self-sensing magnetic bearings are not feasible due to the

K.S. Peterson is with the School of Mechanical Engineering, Purdue University, USA petersks@ecn.purdue.edu

R.H. Middleton is with the Center for Complex Dynamic Systems and Control, University of Newcastle, Australia

J.S. Freudenberg is with Department of Electrical and Engineering and Computer Science, University of Michigan presence of a non-minimum phase (NMP) zero and open right half plane (ORHP) pole in the transfer function from voltage to current. The existence of both a NMP zero and ORHP pole imposes severe limits in the achievable bound on the sensitivity function [3]. However, experimental evidence [9], [10], [11], [12] appears to suggest that the bounds reported by Thibeault and Smith [13] are too restrictive and that better robustness is in fact obtainable using a self-sensing magnetic bearing.

To demonstrate that better robustness is in fact possible, Maslen, Montie, and Iwasaki [6] use a linear periodic (LP) model a self-sensing magnetic bearing rather than a LTI model. The LP component of the model is introduced by the high frequency ripple in the current caused by the PWM drivers. The high frequency ripple appears as a periodic perturbation to the LTI state equations that allows the authors of [6] to extract information about the rotor position from the measurement of current. Based on this LP model of a selfsensing magnetic bearing, the authors of [6] use the methods of Dullerud and Lall [2] to design a LP controller to minimize the peak in the sensitivity function. Maslen, Montie, and Iwasaki [6] then numerically evaluate the norms of the input and output sensitivity functions for their controller. Using this methodology, the authors of [6] are able to demonstrate that the introduction of the periodic perturbation alleviates the robustness issues reported in [13]. Furthermore, the authors of [6] are able to recover the results of [13] in the limit as the magnitude and frequency of the periodic perturbation tend toward zero. While Maslen, Montie, and Iwasaki [6] present an intuitive argument for why the introduction of the periodic perturbation allows for a more robust control design, a rigorous analysis of the system is still needed.

The present paper presents an analysis of the work done by Maslen, Montie, and Iwasaki [6] in order to further investigate why using a LP model of a self-sensing magnetic bearing allows for the constraints found in [13] to be relaxed. This is accomplished by utilizing lifting to convert the LP system to a discrete MIMO LTI system. Once the discrete MIMO LTI system is obtained, established techniques [1] are used to evaluate the robustness limitations of the system and to investigate why they differ from those found in [13].

The use of lifting to investigate the robustness of LP systems has previously been employed in [5], [14]. While Khargonekar, Poolla, and Tannenbaum [5] demonstrate that LP control of LTI systems can be used to improve the achievable gain and phase margins, both they and the authors of [14] have shown that LP control of LTI systems cannot improve the achievable bound on the sensitivity function. The 
main difference between [6] and [5], [14] is that the model is LP and therefore it is probable that LP control will be advantageous, although this remains an open question [5].

The model of the self-sensing magnetic bearing is presented in Sec. II, followed by a review of the results of [6], [13] in Sec. III. Next, an overview of the lifting technique is provided in Sec. IV. Finally, the achievable bounds on the sensitivity function are computed in Sec. V along with a discussion of how the LP nature of the system allows for the constraints found in [13] to be relaxed.

\section{Magnetic Bearing Model}

For the purpose of this paper, the model of the self-sensing magnetic bearing is given by [6], [13]

$$
\begin{aligned}
\frac{d x}{d t} & =A x+\gamma \sin (\omega t) \Delta A x+B u \\
y & =C x+\gamma \sin (\omega t) \Delta C x
\end{aligned}
$$

where

$$
\begin{aligned}
x & =\left[\begin{array}{lll}
g & v & \phi
\end{array}\right]^{\prime} \\
y & =i \\
A & =\left[\begin{array}{ccc}
0 & 1 & 0 \\
0 & 0 & \Phi_{b} \\
\eta \Phi_{b} & 0 & -\eta
\end{array}\right] \\
\Delta A & =\left[\begin{array}{ccc}
0 & 0 & 0 \\
0 & 0 & \Phi_{b} \\
\eta \Phi_{b} & 0 & 0
\end{array}\right] \\
B & =\left[\begin{array}{lll}
0 & 0 & 1
\end{array}\right]^{\prime} \\
C & =\left[\begin{array}{lll}
-\Phi_{b} & 0 & 1
\end{array}\right] \\
\Delta C & =\left[\begin{array}{lll}
-\Phi_{b} & 0 & 0
\end{array}\right]
\end{aligned}
$$

$g$ is the non-dimensional air gap between the rotor and magnetic bearing, $v$ is the non-dimensional velocity of the rotor, $\phi$ is the non-dimensional magnetic flux, $i$ is the nondimensional electrical current, and $u$ is the non-dimensional applied voltage. Values for and definitions of the constants $\Phi_{b}$ and $\eta$ are found in Table I. For $\gamma \neq 0$, this model corresponds to the LP self-sensing magnetic bearing model used in [6] by Maslen, Montie, and Iwasaki. Setting $\gamma=0$, this model corresponds to the LTI model used in [13] by Thibeault and Smith.

\begin{tabular}{c|c|c}
\hline Symbol & Definition & Value \\
\hline$\Phi_{b}$ & non-dim bias flux & 0.288 \\
$\eta$ & ratio of times scales & 0.582 \\
\hline
\end{tabular}

TABLE I

DEFINITIONS AND NUMERICAL VALUES FOR PARAMETERS USED IN THE SELF-SENSING MAGNETIC BEARING MODEL [6].

\section{PREvious ANALYSis of THE SELF-SENSING MAGNETIC BEARING MODEL}

In order to access the feasibility of using a self-sensing magnetic bearing, the authors of both [6] and [13] use the achievable bound on the sensitivity function as a measure of robustness. Given the feedback structures shown in Fig. 2, the input and output sensitivity functions are defined as operators such that

$$
\begin{aligned}
& y=S_{i} w=(I+K P)^{-1} w \\
& u=S_{o} v=(I+P K)^{-1} v
\end{aligned}
$$

respectively, where $P$ is the operator that describes the input/output characteristics of the plant and $K$ is the operator that describes the input/output characteristics of the controller. Given the definitions in Eqns. (3) and (4), the input/output sensitivity functions quantify the response of the system to the input/output divisive uncertainties $\Delta_{i}$ and $\Delta_{o}$ respectively. Thus the achievable bound on the sensitivity function

$$
\left\|S_{i, o}\right\|_{\infty}:=\sup _{\mu \in L_{2} \backslash\{0\}} \frac{\left\|S_{i, o} \mu\right\|_{2}}{\|\mu\|_{2}}
$$

where $L_{2}$ is the set of square integrable signals, is an important measurement of robustness.

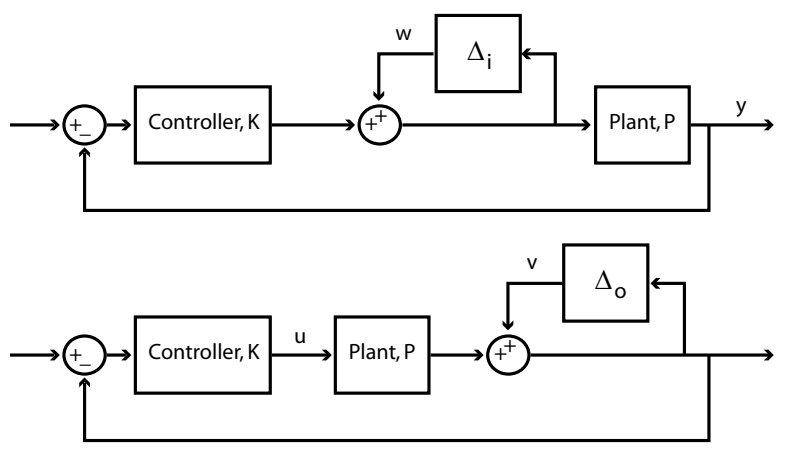

Fig. 2. Generic feedback structure with input divisive uncertainty (top) and output divisive uncertainty (bottom).

Based on the work of [3] and using a LTI model of a self-sensing magnetic bearing, Thibeault and Smith [13] demonstrate that the existence of a NMP zero at $s=0.288$ and ORHP pole at $s=0.242$ in the transfer function from voltage to current imposes a sever limit on the achievable bound on the sensitivity functions, see Table II. As previously stated, these bounds appear to conflict with experimental data given in [9], [10], [11], [12]. To account for this difference, the authors of [6] introduce a periodic perturbation $\gamma \sin (\omega t)$ to model the effects of the high frequency excitation of the PWM drivers used to operate the self-sensing magnetic bearing. Using the techniques developed in [2], the authors of [6] design several controllers to minimize the lower bounds of the sensitivity functions for various values of $\gamma$ and $\omega$. Table II lists the results for $\gamma=0.02$ and $\omega=122$, which is representative of the achieved bounds found in [6].

The authors of [6] argue that the improvements to the input sensitivity function are a direct result of the LP component of the output. Modeled as a LP system, the measured current contains an additional periodic term that is only a function of the air gap between the rotor and magnetic bearing, see 


\begin{tabular}{ccc}
\hline & Results from [13] & Results from [6] \\
\hline$\left\|S_{i}\right\|_{\infty}$ & 11.53 & 1.02 \\
$\left\|S_{o}\right\|_{\infty}$ & 11.53 & 10.50 \\
\hline
\end{tabular}

TABLE II

LOWER BOUNDS ON $\left\|S_{i}\right\|_{\infty}$ AND $\left\|S_{o}\right\|_{\infty}$ FROM [13] AND ACHIEVED BOUNDS ON $\left\|S_{i}\right\|_{\infty}$ AND $\left\|S_{o}\right\|_{\infty}$ FROM [6].

Eqn (2). By extracting this periodic information from the output, the controller is able to determine the air gap between the rotor and magnetic bearing. Since the transfer function from voltage to the air gap is minimum phase, Maslen, Montie, and Iwasaki [6] are able to avoid the limit imposed by the NMP zero in the transfer function from voltage to current. Furthermore, Maslen, Montie, and Iwasaki [6] argue that they are not able to achieve significant improvements in the output sensitivity function since an output disturbance near the frequency of the periodic perturbation $\gamma \sin (\omega t)$ would destroy the ability of the controller to accurately determine the air gap. A similar technique has been utilized to determine rotor position in reluctance motors [7], [4], but as with self-sensing magnetic bearings a complete analysis of the robustness implications is presently lacking in the literature.

To explain why the introduction of the periodic perturbation relaxes the constraints found in [13], the remainder of this paper uses lifting to convert the LP system to a MIMO LTI system. Using the MIMO LTI system, we can compute explicit bounds on the achievable sensitivity functions and investigate why these bounds differ from those found in [13].

\section{DisCRETIZATION AND LifTING}

Explicit bounds for the achievable sensitivity functions of continuous LP systems do not yet exist. As such, the continuous LP model of the self-sensing magnetic bearing is discretized and then lifted to form a LTI system for which explicit bounds do exist. The LP model is discretized using an Euler approximation at the sampling rate

$$
\Delta t=\frac{2 \pi}{\omega N}
$$

where $N$ is the number of desired sample points over one period to form the discrete system

$$
\begin{aligned}
x_{k+1} & =A_{k} x_{k}+B u_{k} \\
y_{k} & =C_{k} x_{k}
\end{aligned}
$$

where

$$
\begin{aligned}
& A_{k}=I+\Delta t(A+\gamma \sin (\omega k \Delta t) \Delta A) \\
& C_{k}=C+\gamma \sin (\omega k \Delta t) \Delta C .
\end{aligned}
$$

The LTI lifted system is given by [8]

$$
\begin{aligned}
x_{(k+1) N} & =F x_{k N}+G v_{k N} \\
q_{k N} & =H x_{k N}+E v_{k N}
\end{aligned}
$$

where

$$
\begin{aligned}
& F=\Phi(N, 0) \\
& G=\left[\begin{array}{lll}
\Phi(N, 1) b_{0} & \Phi(N, 2) b_{1} & \cdots
\end{array}\right. \\
& \left.\Phi(N, N-1) b_{N-2} \quad b_{N-1}\right] \\
& H^{\prime}=\left[\begin{array}{lll}
c_{0}^{\prime} & \Phi(1,0)^{\prime} c_{1}^{\prime} & \cdots
\end{array}\right. \\
& \left.\Phi(N-2,0)^{\prime} c_{N-2}^{\prime} \quad \Phi(T-1,0)^{\prime} c_{N-1}^{\prime}\right] \\
& E^{m n}=\left\{e_{i j}^{m n}\right\} \\
& e_{i j}^{m n}= \begin{cases}c_{i-1} \Phi(i-1, j) b_{j-1} & i>j \\
0 & i \leq j\end{cases}
\end{aligned}
$$

and

$$
\begin{aligned}
& v_{k N}=\left[\begin{array}{llll}
u_{k N} & u_{k N+1} & \cdots & u_{k N+N-1}
\end{array}\right]^{\prime} \\
& q_{k N}=\left[\begin{array}{llll}
y_{k N} & y_{k N+1} & \cdots & y_{k N+N-1}
\end{array}\right]^{\prime} .
\end{aligned}
$$

The matrix $\Phi(i, j)$ is the discrete transition matrix given by

$$
\Phi(i, j)=\Pi_{k=j}^{i-1} A_{k} .
$$

Qualitatively, lifting increases the dimensionality of the input and output vectors and their associated matrices in the state space model such that they contain the inputs and outputs over a single period. In doing so, the new system is LTI with respect to the index $k N$. An important property of the lifted system is that it preserves the norm [5], [14] of the system, i.e. given a discrete system, $P$, and the lifted version, $\hat{P}$,

$$
\|P\|_{\infty}=\|\hat{P}\|_{\infty} .
$$

Due to the property of Eqn. (7) we are able to evaluate the norm based properties of the LP system using the LTI lifted model.

\section{Sensitivity Bounds}

The limitations found in [13] arise due to the signal blocking properties of NMP zeros [3]. By lifting the discrete LP system to form a higher dimensional MIMO system we can make use of the additional inputs/outputs to avoid the blocking properties of the NMP zeros. Mathematically, this appears in terms of the pole/zero input/output directions. The more orthogonal these vectors are to one another, the less the blocking properties of the NMP zeros influence the achievable bounds on the sensitivity functions [1].

Having lifted the LP system to obtain the discrete MIMO state space representation, the input and output sensitivity operators defined in Eqns. (3) and (4) become discrete MIMO transfer functions. As such, the achievable lower bounds for the input and output sensitivity functions are given by [1]

$$
\begin{aligned}
& \left\|S_{i}\right\|_{\infty} \geq \sqrt{\cos ^{2} \angle\left(\eta_{o}, \zeta_{i}\right)\left|\frac{1-p z}{z-p}\right|^{2}+\sin ^{2} \angle\left(\eta_{o}, \zeta_{i}\right)} \\
& \left\|S_{o}\right\|_{\infty} \geq \sqrt{\cos ^{2} \angle\left(\eta_{i}, \zeta_{o}\right)\left|\frac{1-p z}{z-p}\right|^{2}+\sin ^{2} \angle\left(\eta_{i}, \zeta_{o}\right)}
\end{aligned}
$$

where $S_{i}$ is the input sensitivity function, $S_{o}$ is the output sensitivity function, $p$ is the pole outside of the unit circle 
of the lifted system, $z$ is the NMP zero of the lifted system, $\zeta_{i} \in R^{N \times 1}$ is the input zero direction, $\zeta_{o} \in R^{N \times 1}$ is the output zero direction, $\eta_{i} \in R^{N \times 1}$ is the input pole direction, and $\eta_{o} \in R^{N \times 1}$ is the output pole direction. The zero and pole direction vectors are defined as

$$
\begin{gathered}
\zeta_{o}^{\prime}\left(H(z I-F)^{-1} G+E\right)=0 \\
\left(H(z I-F)^{-1} G+E\right) \zeta_{i}=0
\end{gathered}
$$

where $\zeta_{o}^{\prime} \zeta_{o}=1, \zeta_{i}^{\prime} \zeta_{i}=1$, and

$$
\begin{aligned}
\eta_{i} & =\frac{H \nu}{\|H \nu\|_{2}} \\
\eta_{o}^{\prime} & =\frac{v G}{\|v G\|_{2}}
\end{aligned}
$$

where $\nu \in R^{3 \times 1}$ and $v \in R^{1 \times 3}$ are the right and left eigenvectors of $F$ associated with $p$. Table III lists the achievable bounds on both the input and output sensitivity function for two different cases. In each case

$$
\begin{aligned}
\omega & =122 \\
N & =50
\end{aligned}
$$

where $\omega$ is chosen to match the value given in [6]. The value of $\gamma$ is varied to compare our results to those of [6], [13]. Setting $\gamma=0$ reduces the model to the LTI system studied in [13] and it is reassuring to find that the methodology of discretization and lifting produces the same bounds reported in [13]. Next, we let $\gamma=0.2$ to verify the results in [6]. Again, the bounds we have computed match those achieved in [6]. This is not surprising as the methodology used by Maslen, Montie, and Iwasaki [6] employs lifting to apply robust control to LP systems, such as $H_{\infty}$ synthesis [2]. The difference between this paper and [2] is that here we present bounds on the achievable performance whereas [2] presents a methodology for designing a controller to minimize the norm of a LP system.

\begin{tabular}{ccc}
\hline & $\gamma=0$ & $\gamma=0.02$ \\
\hline$\left\|S_{i}\right\|_{\infty}$ & 11.53 & 1.02 \\
$\left\|S_{o}\right\|_{\infty}$ & 11.53 & 10.40 \\
\hline
\end{tabular}

TABLE III

LOWER BOUNDS ON $\|S\|_{\infty}$ FOR VARIOUS VALUES OF $\gamma$.

The location of the discrete pole outside of the unit circle, $p$, and the NMP zero, $z$, of the lifted system are shown in Fig. 3 as a function of $\omega$. In addition the equivalent location in the s-domain of $p$ and $z$ of the lifted system, given by

$$
s=\ln (x) \Delta t
$$

where $x$ is their respective location in the $\mathrm{z}$-domain, are also shown in Fig. 3 as a function of $\omega$. It is reassuring to observe that the lifted system remains both unstable and NMP irrespective of the value of $\omega$. In the $\mathrm{z}$-domain, both the pole and NMP zero approach 1 from above as $\omega$ increases since the sampling time decreases as a function of $\omega$, see Eqn. (6). Somewhat more interesting is that the equivalent locations of the pole and NMP zero in the s-domain are approximately constant with $\omega$. As $\omega$ increases the ORHP pole converges to $s=12.1$ and the NMP zero converges to $s=14.4$. Using these values to compute the continuous time Blaschke product, we find

$$
\left|\frac{p+z}{z-p}\right|=11.52
$$

which is essentially equal to the value of the Blaschke product found in [13] used to find the lower bound on the Sensitivity function.
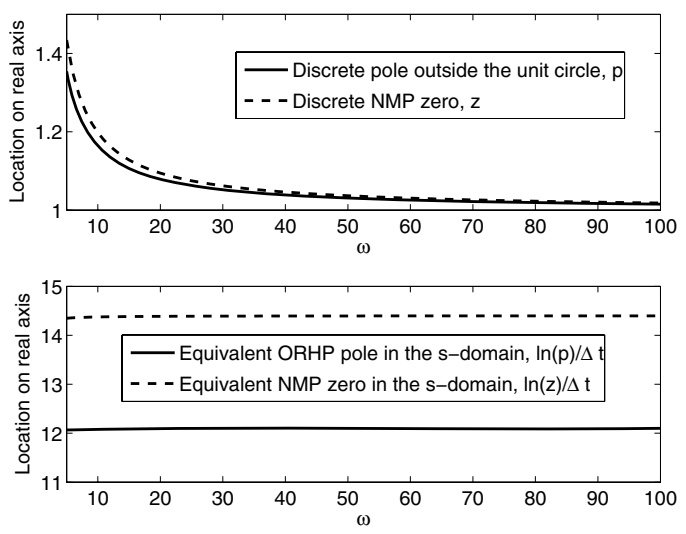

Fig. 3. Locations on the real axis of the discrete pole outside of the unit circle and the NMP zero of the lifted system (Top). Equivalent locations on the real axis in the s-domain of the discrete pole outside of the unit circle and the NMP zero of the lifted system (Bottom).

For an explanation of the improved achievable performance of the lifted system, let us examine the various terms used in Eqns. (8) (9). The terms of interest are the magnitude of the discrete Blaschke product

$$
\left|\frac{1-p z}{z-p}\right|
$$

and the relative angles of the pole/zero input/output directions, $\cos \angle\left(\eta_{o}, \zeta_{i}\right)$ and $\cos \angle\left(\eta_{i}, \zeta_{o}\right)$. Recall that limitations in the sensitivity functions arise due to the existence of NMP zeros and ORHP poles [3] and that their impact is determined by their relative location to one another. The impact of this is encapsulated in the magnitude of the Blaschke product. Thus the further apart the NMP zero and ORHP pole, the smaller the Blaschke product, and thus the smaller its effect. In addition, the relative angles between the pole/zero input/output directions in MIMO systems play an important role as they represent the extent to which the inputs and outputs can be used to "avoid" the effects of the NMP zeros and ORHP poles.

To visualize their effect, the Blaschke product, the $\cos \angle\left(\eta_{o}, \zeta_{i}\right)$, and the $\cos \angle\left(\eta_{i}, \zeta_{o}\right)$ are plotted in Fig. 4 as a function of $\omega$. Both the Blaschke product and $\cos \angle\left(\eta_{i}, \zeta_{o}\right)$ remain relatively constant with changing $\omega$ and thus little 
improvement is observed in the output sensitivity function. In contrast, $\cos \angle\left(\eta_{o}, \zeta_{i}\right)$ approaches zero with increasing $\omega$. Therefore the improvements in the input sensitivity function occur due to the input zero direction and output pole direction becoming orthogonal to one another.
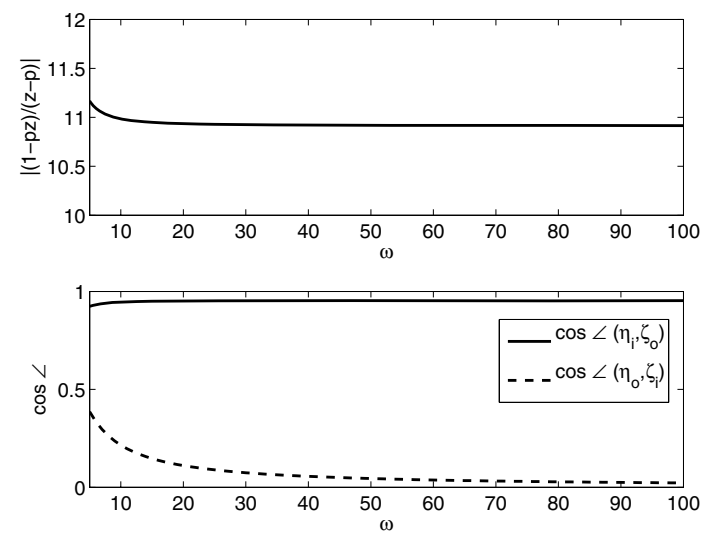

Fig. 4. Blaschke product and angle between zero and pole directions as a function of $\omega$.

To investigate the relative angles of the pole/zero input/output directions further, they are each plotted elementwise in Figs. 5 and 6 for the LP lifted system, $\gamma \neq 0$, and for the LTI lifted system, $\gamma=0$. When the periodic perturbation is introduced the input zero direction, $\zeta_{i}$, output zero direction, $\zeta_{o}$, and input pole direction, $\eta_{i}$, all develop a sinusoidal variation while the output pole direction, $\eta_{o}$, remains essentially constant.
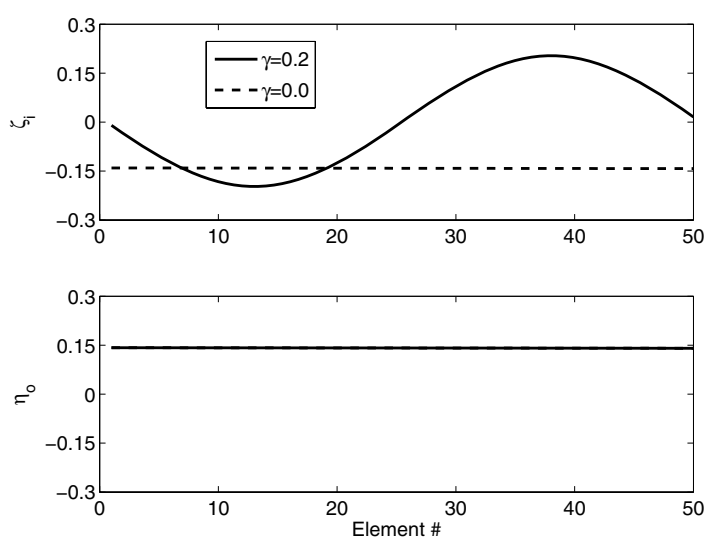

Fig. 5. Pole and zero direction vectors associated with $S_{i}$.

However, it is not the appearance (or lack there of) of a sinusoidal variation in the input/output directions that causes the change in the achievable bound on the sensitivity function. To demonstrate this, the relative angles of the pole/zero input/output directions are each plotted elementwise in Figs. 7 and 8 for the case of $\Delta A=0$ and $\gamma \neq$ 0 which removes the periodic perturbation from the state
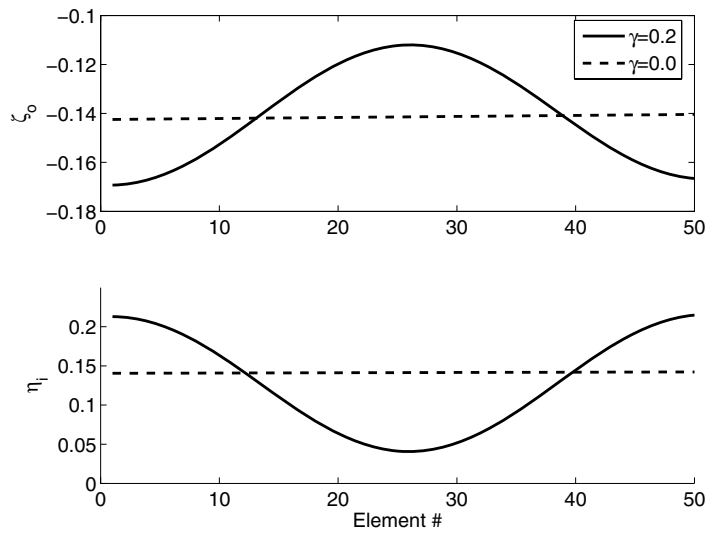

Fig. 6. Pole and zero direction vectors associated with $S_{o}$.

dynamics given in Eqn. (1) but not the output equation. As seen in Fig. 8 the output zero direction, $\zeta_{o}$, does not develop a sinusoidal variation for the case of $\Delta A=0$ while the input pole direction, $\eta_{i}$, is unaffected by setting $\Delta A=0$. Despite this change in the direction vectors the bounds on the sensitivity functions remain unchanged, see Table. IV.
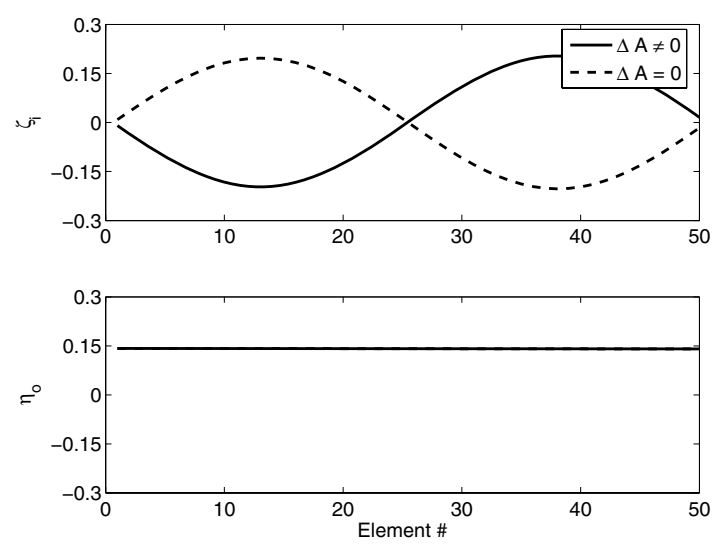

Fig. 7. Pole and zero direction vectors associated with $S_{i}$ with and without including the periodic perturbation on the state dynamics.

If not the appearance of a sinusoidal variation in the input/output directions, what then causes the change in the achievable bound on the sensitivity functions? Recall that the cosine of the angle between two unit vectors is defined as

$$
\cos \angle(\eta, \zeta)=\left|\eta^{\prime} \zeta\right|
$$

Simply put, the cosine of the angle between two unit vectors is the absolute value of the summation of the piece wise multiplication of each element in the two vectors. Therefore the improvement to the input sensitivity function is caused by the fact that $\zeta_{i}$ has a zero mean when the periodic perturbation appears in the output, see Fig. 5. Why the periodic perturbation shifts the mean of $\zeta_{i}$ to zero is unclear. However, the results of Table IV support the claim made by Maslen, 

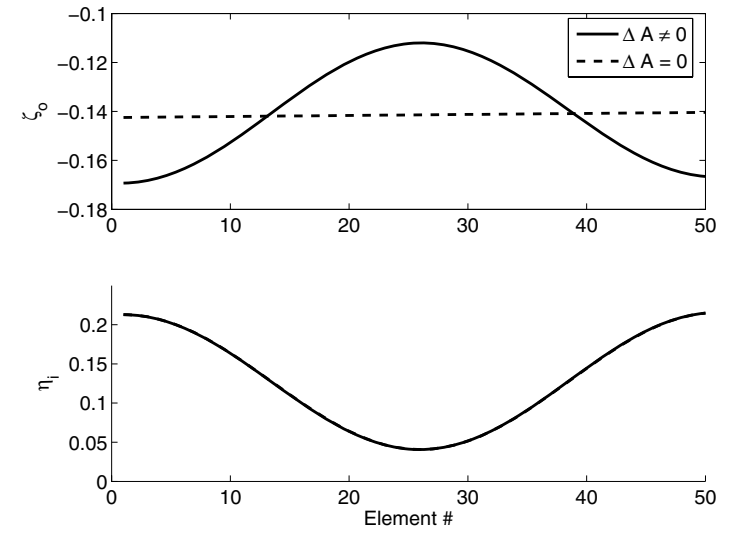

Fig. 8. Pole and zero direction vectors associated with $S_{o}$ with and without including the periodic perturbation on the state dynamics.

Montie, and Iwasaki [6] that the improvement in the input sensitivity function is a result of the periodic perturbation in the output, which contains information about the rotor position in the size and phase of the current ripple. Note that there is little change in the achievable output sensitivity function norm. This implies that there will be at least some classes of disturbances and unmodeled dynamics to which the self-sensing magnetic bearing has high sensitivity. For example, unmodeled measurement time delays could easily destabilize the self-sensing magnetic bearing by causing aliasing or other such distortions to the phase and magnitude of the current ripple. Fortunately, the applied voltage and current measurement are co-located as actuators and sensors, and time delays in the self-sensing magnetic bearing itself are physically impossible. Delays may occur in the measurement electronics but fortunately, these can be accurately controlled by well established electronic design principles.

\begin{tabular}{ccc}
\hline & $\Delta A \neq 0$ & $\Delta A=0$ \\
\hline$\left\|S_{i}\right\|_{\infty}$ & 1.02 & 1.02 \\
$\left\|S_{o}\right\|_{\infty}$ & 10.40 & 10.40 \\
\hline
\end{tabular}

TABLE IV

LOWER BOUNDS ON $\left\|S_{i}\right\|_{\infty}$ AND $\left\|S_{o}\right\|_{\infty}$ FOR $\Delta A \neq 0$ AND $\Delta A=0$.

\section{CONCLUSION}

This paper has presented an analysis of the LP model of a self-sensing magnetic bearing used by Maslen, Montie, and Iwasaki [6] in an attempt to gain insight into why it appears to contradict the results published by Thibeault and Smith [13]. By utilizing lifting to convert the LP model to a discrete MIMO LTI model, it was shown that improvements in the achievable bound on the sensitivity function is a result of the orthogonality of the pole/zero input/output direction vectors due to the periodic nature of the output. While strengthening the claims made by Maslen, Montie, and Iwasaki [6], more work is still required to better understand how the controller should exploit the periodic nature of the system to improve robustness and when similar techniques can be applied to other systems.

\section{REFERENCES}

[1] J. Chen and C. Nett, "Sensitivity integrals for multivariable discretetime systems," Automatica, vol. 31, no. 8, pp. 1113-1124, Aug. 1995.

[2] G. Dullerud and S. Lall, "A new approach for analysis and synthesis of time-varying systems," IEEE Transactions on Automatic Control, vol. 44, no. 8, pp. 1486-1497, Aug. 1999.

[3] J. Freudenberg and D. Looze, "Right half plane poles and zeros and design tradeoffs in feedback systems," IEEE Transactions on Automatic Control, vol. 30, no. 6, pp. 555-565, june 1985.

[4] M. Jovanovic, R. Betz, and D. Platt, "Sensorless vector controller for a synchronous reluctance motor," IEEE Transactions of Industry Applications, vol. 34, no. 2, pp. 346-354, 1998.

[5] P. Khargonekar, K. Poolla, and A. Tannenbaum, "Robust control of linear time-invariant plants using periodic compensation," IEEE Transactions on Automatic Control, vol. 30, no. 11, pp. 1088-1096, Nov. 1985.

[6] E. Maslen, D. Montie, and T. Iwasaki, "Robustness limitations in self-sensing magnetic bearings," to appear in the ASME Journal of Dynamic Systems, Measurement, and Control, 2005.

[7] T. Matsuo and T. Lipo, "Rotor position detection scheme for synchronous reluctance motor based on current measurements," IEEE Transactions of Industry Applications, vol. 31, no. 4, pp. 860-868, 1995.

[8] R. Meyer and C. Burrus, "A unified analysis of multirate and periodically time-varying digital filters," IEEE Transactions on Circuits and Systems, vol. 22, no. 3, pp. 162-168, Mar. 1975.

[9] T. Mizuno, T. Ishii, and K. Araki, "Self-sensing magnetic suspension using hysteresis amplifiers," Control Engineering Practice, vol. 6, pp. 1133-1140, 1998.

[10] D. Montie, "Performance limitations and self-sensing magnetic bearings," Ph.D. dissertation, University of Virginia, 2003.

[11] M. Noh and E. Maslen, "Self-sensing magnetic bearings using parameter estimation," IEEE Transactions on Instrumentation and Measurement, vol. 46, no. 1, pp. 45-50, Feb. 1997.

[12] K. Sivadasan, "Analysis of self-sensing active magnetic bearings working on inductance measurement principle," IEEE Transactions on Magnetics, vol. 32, no. 2, pp. 329-334, Mar. 1996.

[13] N. Thibeault and R. Smith, "Magnetic bearing measurement configurations and associated robustness and performance limitations," ASME Journal of Dynamic Systems, Measurement, and Control, vol. 124, pp. 589-598, Dec. 2002.

[14] C. Zhang, J. Zhang, and K. Furuta, "Analysis of $h_{2}$ and $h_{\infty}$ performance of discrete periodically time-varying controllers," Automatica, vol. 33, no. 4, pp. 619-634, 1997. 